\title{
A derivation of thermodynamic principles
}

\section{J R Sanmartín}

\author{
ETS Ingenieros Aeronáuticos, Universidad Politécnica, 28040 Madrid, Spain
}

Received 14 November 1995

\begin{abstract}
A detailed macroscopic description as in continuum mechanics, and just the concept of microstate, have been used to derive thermodynamics from mechanics. In opposition to statistical physics, the derivation lays emphasis on a definite prescription for macrostates (and non-equilibrium entropy), and uses basic features of the macrostate concept: complementary descriptions, involving either conservative and additive quantities or densities; scale-free character; reference to finite velocities and regions distant in space, thus introducing time indirectly. On the other hand, the derivation keeps the particle substratum (limit of number of particles $N \rightarrow \infty$ taken at fixed densities), and makes no ergodic-type considerations.
\end{abstract}

\section{Introduction}

Expositions of principles differ in thermodynamics as in no other part of physics (Lewins 1985, Gyftopoulos and Beretta 1991, Baierlein 1994). As often observed, the traditional macroscopic approach is neither natural nor simple. One option gaining ground in the last decades has been to mix the traditional and statistical physics approaches (Landau and Lifshitz 1969, Bauman 1992). This requires a detailed study of microstates that usually leaves the concept of macrostate vague or narrow.

To deal with thermodynamics itself - a macroscopic theory anyway-we propose shifting emphasis in the mixed approach, to give a definite prescription for macrostates, as in continuum mechanics; the prescription involves basic mechanical features occasionally made explicit in the literature (Landau and Lifshitz 1969, Callen 1974). It is essential, however, to keep (just) the concept of microstate, so as to allow a definite prescription for non-equilibrium entropy, too. Otherwise one would be left with continuum mechanics, which takes thermodynamics as input (Batchelor 1967), or (the heavily axiomatic) continuum thermodynamics, which is just axiomatics (Truesdell 1969).

The modified mixed approach here presented lays emphasis on the additive and conservative character of quantities used in the macroscopic description, and on the fact that such description, being characterized
Resumen. Se introduce la detallada descripción macroscópica de la mecánica de medios continuos, junto al simple concepto de microestado, para derivar la termodinámica a partir de la mecánica. En oposición a la física estadística, la derivación enfatiza una prescripción precisa para macroestados (y la entropía fuera del equilibrio) y utiliza rasgos básicos del concepto de macroestado: descripciones complementarias que involucran densidades ó cantidades conservativas y aditivas; ausencia de escala absoluta; referencia a velocidades finitas y regiones distantes en el espacio, lo que reintroduce el tiempo indirectamente. Por el contrario, la derivación mantiene la estructura de partículas del medio (límite de número de partículas $N \rightarrow \infty$ tomado a densidad constante) y evita consideraciones de tipo ergódico.

by densities (that are kept fixed in the formal thermodynamic limit of an infinite number of particles), is scale-free. Use is also made of the fact that the macrostate concept introduced, involving both finite velocities and distant spatial regions, brings in time indirectly. Our approach then provides a direct derivation of thermodynamic principles by way of a verified ansatz. Alternative derivations proceed from statistical physics, and are concerned with weak forms of the old ergodic hypothesis; in spite of profound advances in the analysis of dynamical systems (Bernouilli flows, Hamiltonian chaos (see Grandy 1987)), such approaches remain largely postulatory. The convenience of a foundation of thermal physics that entirely ignores ergodic questions has been discussed in the past (Khinchin 1949). For twenty years, the author has used a simplified form of the present exposition to make smooth to students the transition from mechanics to thermodynamics.

In the next section we establish the formal concept of a macrostate. In sections 3 and 4 we prove the existence and properties of an equilibrium macrostate for ideally set up systems. In section 5 we extend the results to a broader class of situations. Conclusions are briefly restated in the final section. 


\section{The macrostate concept}

The full classical description of a body having a very large number $N$ of particles, assumed to be equalmass points for simplicity, requires solving $N$ coupled Newton equations. Given the interaction law, any initial complete microstate (positions $\bar{r}_{j}$ and velocities $\bar{v}_{j}$ for $j=1,2, \ldots, N$ ) would determine the entire behaviour of the system. We assume here that one has given up on such microscopic descriptions.

In continuum mechanics a macroscopic body is viewed, instead, as a large collection of volume elements $\delta V_{\alpha}(\alpha=1,2, \ldots)$, which are thus small if compared with the body itself but still contain a large number of particles $\delta N_{\alpha}$. The description takes a certain middle course: each element, located at a position $\bar{r}_{\alpha}$, is considered as neither a mass point nor a full subsystem of $\delta N_{\alpha}$ mass points: only a few particular quantities, $\left\{\delta \chi_{\alpha}\right\}$, dependent on the motion of the $\delta N_{\alpha}$ particles, are studied for each element. These quantities must satisfy two conditions:

(a) $\delta \chi_{\alpha}$ should be conserved in the special case of a closed subsystem (a subsystem that neither exchanges particles nor interacts with its external environment). The dynamical evolution of $\delta \chi_{\alpha}$ would then follow the trivial law $\delta \chi_{\alpha}(t)=$ constant. Furthermore, in the general case the evolution would depend on the external environment of the element and might possibly be determined without looking inside it, i.e. without reverting to the microscopic description. This would be impossible for quantities that may change in closed subsystems.

(b) $\delta \chi_{\alpha}$ should be additive, that is, for neighbouring, non-overlapping elements $\alpha$ and $\beta$, one should have $\delta \chi_{(\alpha, \beta)}=\delta \chi_{\alpha}+\delta \chi_{\beta}$, where $(\alpha, \beta)$ is the joint element of volume $\delta V_{(\alpha, \beta)}=\delta V_{\alpha}+\delta V_{\beta}$. This is because the macroscopic description requires the densities $\delta \chi_{\alpha} / \delta V_{\alpha}$ to be non-arbitrary, shape and scale of volume elements being to a large extent arbitrary. Since the neighbouring elements $\alpha$ and $\beta$ and the joint element $(\alpha, \beta)$ are (approximately) in the same location, one should have

$\frac{\delta \chi_{\alpha}}{\delta V_{\alpha}}=\frac{\delta \chi_{\beta}}{\delta V_{\beta}}=\frac{\delta \chi_{(\alpha, \beta)}}{\delta V_{(\alpha, \beta)}} \longrightarrow \frac{\delta \chi_{\alpha}+\delta \chi_{\beta}}{\delta V_{\alpha}+\delta V_{\beta}}=\frac{\delta \chi_{(\alpha, \beta)}}{\delta V_{(\alpha, \beta)}}$,

wherefrom additivity follows. For (a macroscopic part of) the body, an additive quantity has a value $\sum_{\alpha} \delta \chi_{\alpha}$ irrespective of the partition in volume elements.

Three quantities, stemming from the homogeneity of space and time and the isotropy of space, satisfy condition (a) with all generality: momentum $\left(\delta \bar{P}_{\alpha}\right)$, angular momentum about some fixed point $A\left(\delta \bar{L}_{A \alpha}\right)$, and energy $\left(\delta E_{\alpha}\right.$, sum of kinetic energy and potential energy of internal forces). One must add here $\delta N_{\alpha}$, or $\delta M_{\alpha}=m \delta N_{\alpha}$ ( $m \equiv$ particle mass), because volume, rather than mass, defines a subsystem. Mass, momentum, angular momentum and kinetic energy, being given by single-index sums (e.g. $\delta \bar{P}_{\alpha}=\sum \bar{P}_{j}$, $j \in \alpha)$, clearly satisfy condition (b) too. Since the requirement $\delta N_{\alpha} \gg 1$ implies $\delta V_{\alpha}^{1 / 3} \gg$ particle size, the potential energy is also additive for real systems with short-range forces; further, charge screening and overall neutrality makes plasmas similar to bodies with shortrange forces, as regards thermodynamics. (The case of gravitationally-bound systems is radically different, and merits separate consideration (Sanmartín 1995).) Hence, in the above discussion, the symbol $\chi$ would represent $M$ (or $N$ ), $\bar{P}, \bar{L}_{A}$ and $E$.

Instead of $\delta \bar{L}_{A \alpha}$ and $\delta E_{\alpha}$ one may actually use the quantities

$$
\begin{aligned}
& \delta \bar{L}_{i \alpha} \equiv \delta \bar{L}_{A \alpha}-\left(\bar{r}_{\alpha}-\bar{r}_{A}\right) \wedge \delta \bar{P}_{\alpha}, \\
& \delta E_{i \alpha} \equiv \delta E_{\alpha}-\delta P_{\alpha}^{2} / 2 \delta M_{\alpha},
\end{aligned}
$$

which are the angular momentum of the subsystem about its centre of mass, and the energy in its centre-of-mass frame (internal energy), respectively. Furthermore, one may ignore $\delta \bar{L}_{i \alpha}$, which is smaller than $\left(\bar{r}_{\alpha}-\bar{r}_{A}\right) \wedge \delta \bar{P}_{\alpha}$ by a factor of the order of the small ratio $\delta V_{\alpha}^{1 / 3} /$ (the characteristic length of the body). For this reason, a state (macrostate) in continuum mechanics is completely determined by giving three quantities, $\delta M_{\alpha}, \delta P_{\alpha}$, and $\delta E_{i \alpha}$, and the volume element $\delta V_{\alpha}$, at each position $\bar{r}_{\alpha}$. Alternatively, one may introduce three densities,

$$
\frac{\delta M_{\alpha}}{\delta V_{\alpha}} \equiv \rho\left(\bar{r}_{\alpha}\right), \quad \frac{\delta \bar{P}_{\alpha}}{\delta V_{\alpha}} \equiv \rho \bar{v}\left(\bar{r}_{\alpha}\right), \quad \frac{\delta E_{i \alpha}}{\delta V_{\alpha}} \equiv \rho e_{i}\left(\bar{r}_{\alpha}\right),
$$

the macrostate being now given by point functions $\rho(\bar{r})$, $\bar{v}(\bar{r})$ and $e_{i}(\bar{r})$ throughout the body; $\bar{v}$ and $e_{i}$ are the specific momentum and internal energy. The basic equations of continuum mechanics deal with densities $\rho, \bar{v}$ and $e_{i}$ (note that $\delta L_{i \alpha} / \delta V_{\alpha} \sim \delta V_{\alpha}^{1 / 3}$ would vanish in the continuum description, $\delta V_{\alpha} \rightarrow 0$ ). Here, however, we are only interested in the (definite) concept of macrostate, which, together with the concept of microstate, makes deriving thermodynamics possible.

\section{The basis of thermodynamics}

The complete information represented by any microstate (the values of all $\bar{r}_{j}$ and $\bar{v}_{j}$ ) determines a single corresponding macrostate (how many particles $\delta N_{\alpha}$ are in each element $\delta V_{\alpha}$, and so on). On the other hand, the limited information of a macrostate clearly determines not one but a large number $v$ of (accessible) microstates, which have this same corresponding macrostate. We may explicitly write

$v($ macrostate $) \equiv v\left[\left\{\delta N_{\alpha}\right\},\left\{\delta V_{\alpha}\right\},\left\{\delta E_{i \alpha}\right\},\left\{\delta \bar{P}_{\alpha}\right\}\right]$.

Naturally, neither microstates nor macrostates can be counted in classical physics. The number $v$ is here a normalized measure (volume) for point sets in the $6 N$-dimensional space of positions and velocities (or momenta, for convenience). Thermodynamics, anyway, is generically insensitive to the choice of measure, because no phase-space averages are performed and because of the particular character of an exceptional macrostate, as shown later in this section. Thermodynamics rests upon a few basic features 
underlying the function $v$ : quantities of macroscopic interest satisfy conditions (a) and (b); the description, being characterized by densities, is scale-free; $N$ is very large, the formal (thermodynamic) limit $N \rightarrow$ $\infty$ being taken at fixed average densities; both finite velocities and distant spatial regions are involved in the description. These features are used in the following.

The function $v$ may be studied indirectly for a broad class of macrostates by considering a simpler, fundamental problem: given a body of volume $V$, find the number $\Gamma$ of microstates compatible with only the overall values $M$ (or $N), E$ and $P \equiv|\bar{P}|$ ( $\Gamma$ being clearly independent of any direction). In the centre-ofmass frame for the body, characterized by the values of $E_{*}=E-P^{2} / 2 M$ and $\bar{P}_{*}=0$, we will have $\Gamma\left(N, V, E_{*}\right.$, $\left.\bar{P}_{*}=0\right)$; as proved in section 4 , one may actually ignore condition $\bar{P}_{*}=0$, thus writing $\Gamma\left(N, V, E_{*}\right)$. Note that many macrostates will be also compatible with the above overall values, a number of microstates $v$ among the total $\Gamma$ being accessible to each macrostate. We now make the ansatz, also proved in section 4 , that the function $v$ presents a maximum value, $v_{e}$, at one particular macrostate called thermodynamic equilibrium, the maximum being overwhelmingly sharp, $v_{e} / \Gamma \simeq 1$ $\left(v_{e} / \Gamma \rightarrow 1\right.$ in the thermodynamic limit). This has several fundamental consequences.

First, consider a macroscopic body that is a closed system, keeping constant values of $N, V, E$ and $\bar{P}$. Generically, then, as the system wanders among its $\Gamma$-compatible microstates, it will be found in the equilibrium macrostate during an overwhelming fraction of any sufficiently long period of time. A variant of this result concerns a system that gets closed while in a microstate inaccessible to the equilibrium for the overall conditions prevailing at that time-and afterwards. Generically, again, the body will approach equilibrium drifting through macrostates with increasing $v$, which proves to be a continuous function of the macrostate, itself a continuous function of the microstate.

Next, note that the approach to equilibrium involves a transport of mass, energy and momentum among distant spatial regions, the time required for that process thus increasing with the size of the body. Note, further, that any volume element or subsystem might itself be considered as a macroscopic system (its size being arbitrary within a broad range, it could, in principle, be divided into a large number of subelements, each still containing a great many particles). This allows us to introduce the number of microstates $v_{\alpha}$ accesible to the macrostate of the subsystem, the number $v$ for the whole body being obtained from the Cartesian product of microstate sets,

$$
v=\prod_{\alpha} v_{\alpha}
$$

it also brings back the fundamental problem at subsystem level, the values $\delta N_{\alpha}, \delta V_{\alpha}, \delta E_{i \alpha} \equiv \delta E_{* \alpha}$ and $\delta \bar{P}_{\alpha}$, determining the number $\Gamma\left(\delta N_{\alpha}, \delta V_{\alpha}, \delta E_{i \alpha}\right)$. Note, last, that the body is much larger than its subsystems, and so is the time it takes to approach equilibrium. We then conclude that, as the body drifts among its nonequilibrium macrostates, slowly redistributing mass, energy and momentum, each evolving subsystem will keep near its own local equilibrium for the instantaneous values $\delta N_{\alpha}, \delta \bar{P}_{\alpha}$ and $\delta E_{i \alpha}$. We can, therefore, write

$$
v_{\alpha} \simeq v_{\alpha e} \simeq \Gamma_{\alpha} \equiv \Gamma\left(\delta N_{\alpha}, \delta V_{\alpha}, \delta E_{i \alpha}\right) .
$$

Equations (2) and (3) thus yield an explicit expression to use in (1),

$$
v(\text { macrostate }) \simeq \prod_{\alpha} \Gamma\left(\delta N_{\alpha}, \delta V_{\alpha}, \delta E_{i \alpha}\right) .
$$

Finally, note that equation (4) involves just one function of three arguments, even though $v$ depends on a huge number of variables. Equations (2) and (3) further show that $\ln \Gamma$ is additive. Then, by requiring $\Gamma$ to satisfy condition $\ln \Gamma_{(\alpha, \beta)}=\ln \Gamma_{\alpha}+\ln \Gamma_{\beta}$ for neighbouring elements $\alpha$ and $\beta$, as in section 2 , and using the additivity of $N, V$ and $E_{i}$, one may trivially show that $\Gamma$ must take the form

$$
\Gamma=\left[f\left(V / N, E_{*} / N\right)\right]^{N} .
$$

\section{Properties of equilibrium}

We now use (5) to show, first, why we may ignore condition $\bar{P}_{*}=0$ : in the thermodynamic limit, only vanishing values of $P_{*}^{2} / 2 M E_{*}$ make a contribution to $\Gamma\left(N, V, E_{*}\right)$. To prove this, assume $\bar{P}_{*} \neq 0$, move to the new centre-of-mass frame, and again ignore the new condition, $\bar{P}_{* *}=0$, in $\Gamma\left(N, V, E_{* *}=\right.$ $\left.E_{*}-P_{*}^{2} / 2 M, \bar{P}_{* *}=0\right)$. Then, using (5) and taking $P_{*}^{2} / 2 M E_{*}^{*}$ to be small, we have

$$
\begin{gathered}
\frac{\left[f\left(\frac{V}{N}, \frac{E_{*}}{N}-\frac{P_{*}^{2}}{2 M N}\right)\right]^{N}}{\left[f\left(\frac{V}{N}, \frac{E_{*}}{N}\right)\right]^{N}} \simeq\left[1-\frac{P_{*}^{2}}{2 M N} \frac{\partial \ln f}{\partial\left(E_{*} / N\right)}\right]^{N} \\
\simeq \exp \left[-N \frac{P_{*}^{2}}{2 M E_{*}} \frac{E_{*}}{N} \frac{\partial \ln f}{\partial\left(E_{*} / N\right)}\right] .
\end{gathered}
$$

Since $f$ and its derivatives remain finite along with its arguments (and the average densities) as $N \rightarrow$ $\infty,\left(E_{*} / N\right) \partial \ln f / \partial\left(E_{*} / N\right)$ is a finite dimensionless number. Thus, assuming that condition

$$
\frac{\partial \ln f}{\partial\left(E_{*} / N\right)} \equiv \frac{\partial \ln \Gamma}{\partial E_{*}}>0
$$

is satisfied, only the values $P_{*}^{2} / 2 M E_{*} \sim 1 / N$ or smaller make up a non-negligible part of $\Gamma$.

We may next use (5) to verify the ansatz concerning the equilibrium macrostate, and to determine its properties. Consider the set of macrostates with $\frac{1}{2} N$ particles and $\frac{1}{2} E_{*} \pm \Delta E$ energies in each of the two half-volumes $\frac{1}{2} V$. Let $\sum \nu(\Delta E)$ be the number of microstates accessible to this set, among the total $\Gamma$. We would have

$$
\begin{gathered}
\sum v(\Delta E)=\Gamma\left(\frac{N}{2}, \frac{V}{2}, \frac{E_{*}}{2}+\Delta E\right) \\
\times \Gamma\left(\frac{N}{2}, \frac{V}{2}, \frac{E_{*}}{2}-\Delta E\right),
\end{gathered}
$$


and, assuming $\Delta E / E_{*}$ small and using (5), arrive at

$$
\begin{gathered}
\frac{\sum v(\Delta E)}{\Gamma\left(N, V, E_{*}\right)} \simeq\left[1+\left(\frac{2 \Delta E}{N}\right)^{2} \frac{\partial^{2} \ln f}{\partial\left(E_{*} / N\right)^{2}}\right]^{N / 2} \\
\simeq \exp \left[N\left(\frac{\Delta E}{E_{*}}\right)^{2} 2\left(\frac{E_{*}}{N}\right)^{2} \frac{\partial^{2} \ln f}{\partial\left(E_{*} / N\right)^{2}}\right] .
\end{gathered}
$$

Arguing as for equation (6), we conclude that if condition

$$
\partial^{2} \ln f / \partial\left(E_{*} / N\right)^{2}=N \partial^{2} \ln \Gamma / \partial E_{*}^{2}<0
$$

is satisfied, only the values $\left(\Delta E / E_{*}\right)^{2} \sim 1 / N$ or smaller make up a non-negligible part of $\Gamma$. For such low $\Delta E$, the values $E_{*} / N$ and $\left(E_{*} \pm 2 \Delta E\right) / N$ in the half-volumes represent, in the thermodynamic limit, the same set of macrostates (same densities).

A similar result applies to macrostates with $\frac{1}{2} E_{*}$ energies and $\frac{1}{2} N \pm \Delta N$ particles in each of the two volumes $\frac{1}{2} V$. The exponent in (8) is $2(\Delta E)^{2} \partial^{2} \ln \Gamma / \partial E_{*}^{2}$. Since (5) can be rewritten as $\Gamma=\left[\varphi\left(N / E_{*}, V / E_{*}\right)\right]^{E_{*}}$ for some appropriate function $\varphi$, we find immediately an equation similar to (8),

$$
\begin{gathered}
\frac{\sum v(\Delta N)}{\Gamma\left(N, V, E_{*}\right)} \simeq \exp \left[2(\Delta N)^{2} \frac{\partial^{2} \ln \Gamma}{\partial N^{2}}\right] \\
=\exp \left[N\left(\frac{\Delta N}{N}\right)^{2} 2 \frac{N}{E_{*}} \frac{\partial^{2} \ln \varphi}{\partial\left(N / E_{*}\right)^{2}}\right],
\end{gathered}
$$

where instead of $(7 b)$ we require condition

$$
\partial^{2} \ln \Gamma / \partial N^{2}<0 .
$$

Finally, equation (6) may also be used to show that only macrostates with negligible $\bar{P}$ in either half-volume make a finite contribution to $\Gamma\left(N, V, E_{*}\right)$.

We can now proceed with each half-volume as with the entire volume $V$, with similar results. This could be repeated for a sequence $\frac{1}{4} V, \frac{1}{8} V, \ldots$, ending at some appropriate volume element $\delta V=V / 2^{n}$, for a range of moderately large integers $n$; only macrostates with $2^{-n} N$ particles, $2^{-n} E_{*}$ energies and zero momenta in each of the $2^{n}$ elements will contribute non-negligibly to the total $\Gamma$. The additivity of all three quantities, $N$, $E_{*}$ and $V$, allows one to extend these results to volume elements of any shape and size (within a broad range). The final conclusions are: (1) The maximum of $v$, at the equilibrium macrostate, is overwhelmingly sharp, as advanced; (2) at equilibrium $\delta N_{\alpha} / \delta V_{\alpha}$ and $\delta E_{i \alpha} / \delta V_{\alpha}$ reach uniform values $N / V$ and $E_{*} / V$ throughout the body, and (3) there is no macroscopic motion $\left(\delta \bar{P}_{\alpha} / \delta N_{\alpha}=0\right.$ for every $\left.\alpha\right)$, the energy being entirely internal, $E_{*}=E_{i}$.

\section{Non-closed systems}

The entropy of a general macrostate is defined as

$$
\begin{aligned}
\ln v & =\sum_{\alpha} \ln v_{\alpha} \simeq \sum_{\alpha} \ln \Gamma_{\alpha} \\
& =\sum_{\alpha} \delta N_{\alpha} \ln f\left(\delta V_{\alpha} / \delta N_{\alpha}, \delta E_{i \alpha} / \delta N_{\alpha}\right) .
\end{aligned}
$$

Entropy is thus additive, like $N, V, E_{i}$ or $\bar{P}$, but nonconservative: it grows to its equilibrium maximum, given by

$\ln v_{e}=\sum_{\alpha} \delta N_{\alpha} \ln f\left(V / N, E_{i} / N\right)=\ln \Gamma\left(N, V, E_{i}\right)$.

All the thermodynamic properties of a system relate to the equilibrium entropy $\ln \Gamma\left(N, V, E_{i}\right)$, written as $S\left(N, V, E_{i}\right)$ for brevity. Local values of intensive quantities, defined as ratios or differential ratios of additive quantities, like densities, entropy per particle $S / N$, or the derivatives

$\left.\frac{\partial S}{\partial E_{i}}\right|_{N, V} \equiv \frac{1}{T},\left.\quad \frac{\partial S}{\partial V}\right|_{N, E_{i}} \equiv \frac{p}{T},\left.\quad \frac{\partial S}{\partial N}\right|_{V, E_{i}} \equiv \frac{-\mu}{T}$,

can be written in terms of two other local intensive quantities, $\delta N_{\alpha} / \delta V_{\alpha}$ and $\delta E_{i \alpha} / \delta V_{\alpha}$ in particular, and will thus be uniform throughout a body at equilibrium. Conditions (7a)-(7c) require $T$ (temperature), $\partial E_{i} /\left.\partial T\right|_{N, V}$ (heat capacity) and $-\partial p /\left.\partial V\right|_{N, T}$ to be positive (Landau and Lifshitz 1969). Additive quantities are clearly extensive, that is, at equilibrium; they are proportional to the volume considered.

An exposition of thermodynamics would now go on to consider more complex systems and problems (twobody equilibrium, mixtures, phases, chemical reactions, cycles). Instead, we discuss here a number of points concerning the basic closed-body concept.

(i) If a system is nearly, but not fully, closed (that is, $\mathrm{d} \ln N / \mathrm{d} t, \mathrm{~d} \ln V / \mathrm{d} t$ and $\mathrm{d} \ln E_{i} / \mathrm{d} t$ are non-vanishing but small as compared with the inverse time needed to approach equilibrium), it must keep near equilibrium at all times. One will have, in particular, $\ln v(t) \simeq$ $S\left[N(t), V(t), E_{i}(t)\right]$ and

$$
\frac{\mathrm{d} S}{\mathrm{~d} t}=-\frac{\mu}{T} \frac{\mathrm{d} N}{\mathrm{~d} t}+\frac{p}{T} \frac{\mathrm{d} V}{\mathrm{~d} t}+\frac{1}{T} \frac{\mathrm{d} E_{i}}{\mathrm{~d} t} .
$$

This can be (surprisingly) read as an energy balance,

$$
\mathrm{d} E_{i}=T \mathrm{~d} S-p \mathrm{~d} V+\mu \mathrm{d} N .
$$

Equation (9) could now lead to the notion of heat, $1 / T$ as its integrating factor, and entropy growth under heat flow from high to low temperatures.

(ii) Equation (9) shows that $p$ is pressure exerted at the surface of the body. Prescribing the volume of a closed body amounts to setting up an infinite potential barrier at the surface, as a special external field; if the barrier moves in slowly so as to keep near-equilibrium, the work done by uniform pressure $p$ would be $-p \mathrm{~d} V$, modifying $E_{i}$ independently of any particle or heat exchange. Note that the ideal concept of a macroscopic 
body as a closed system, introduced in sections 2 and 3 in such a way that a fundamental problem reappeared for subsystems, has ended up requiring a pressure that is incompatible with one characteristic of closed systems (a constant value of $\bar{P}$ ), because fixing an ideal potential barrier can ensure conservation of energy but not of momentum. As we have seen, however, the ratio $\Gamma\left(N, V, E_{*}, \quad \bar{P}_{*}=0\right) / \Gamma\left(N, V, E_{*}\right)$ approaches unity in the thermodynamic limit, a condition of vanishing momentum being therefore equivalent to just ignoring momentum conservation. This reflects on the pressure being uniform at equilibrium, thus exerting no net force on the surface of the body and preserving the vanishing $\bar{P}_{*}$ value; it also ensures that the energy can remain fully internal during the evolution represented by the balance equation (9).

Let us use equation (4) instead of (5), to derive the equilibrium properties of section 4 in a different manner (which fails, however, to prove the limit $v_{e} / \Gamma \rightarrow$ 1 for $N \rightarrow \infty)$. Equilibrium of a closed system corresponds to a maximum of $\prod_{\alpha} \Gamma\left(\delta N_{\alpha}, \delta V_{\alpha}, \delta E_{i \alpha}\right)$ subject to conditions $\sum_{\alpha} \delta N_{\alpha}=N, \sum_{\alpha} \delta \bar{P}_{\alpha}=\bar{P}_{*} \equiv 0$, and $\sum_{\alpha}\left(\delta E_{i \alpha}+\delta P_{\alpha}^{2} / 2 m \delta N_{\alpha}\right)=E_{*}$, with all volume elements fixed. This is equivalent to requiring maximum $\Gamma_{\beta} \Gamma_{\gamma}$ for any two subsystems $\beta$ and $\gamma$, with

$$
\begin{aligned}
& \delta V_{\beta}, \quad \delta V_{\gamma}, \quad \delta N_{\beta}+\delta N_{\gamma}, \quad \delta \bar{P}_{\beta}+\delta \bar{P}_{\gamma} \\
& \delta E_{i \beta}+\delta E_{i \gamma}+\delta P_{\beta}^{2} / 2 m \delta N_{\beta}+\delta P_{\gamma}^{2} / 2 m \delta N_{\gamma},
\end{aligned}
$$

and values in all other subsystems, fixed. One then finds $\delta \bar{P}_{\beta} / \delta N_{\beta}=\delta \bar{P}_{\gamma} / \delta N_{\gamma}, T_{\beta}=T_{\gamma}$ and $\mu_{\beta}=\mu_{\gamma}$. Hence, $\delta \bar{P}_{\alpha} / \delta N_{\alpha}$ is uniform (and vanishing because of the global condition $\sum_{\alpha} \delta \bar{P}_{\alpha}=0$ ) and $T$ and $\mu$, and thus all intensive quantities, are uniform too, as in section 4 . If one now retakes these calculations with the momentum condition ignored, identical results follow.

(iii) The preceding discussion may be modified to take into account the conservation of angular momentum of a closed body $\left(\bar{L}_{A *}\right)$, as an additional condition, up to here ignored in our study of equilibrium. Since the center of mass of the body is at rest, it can be taken as point $\mathrm{A}$ and be placed at the origin. Using $\delta \bar{L}_{i \alpha} \simeq 0$, the new condition reads $\sum_{\alpha} \bar{r}_{\alpha} \wedge \delta \bar{P}_{\alpha}=\bar{L}_{A *}$; alternatively, there is a new quantity,

$$
\bar{r}_{\beta} \wedge \delta \bar{P}_{\beta}+\bar{r}_{\gamma} \wedge \delta \bar{P}_{\gamma},
$$

to be kept fixed in the determination of maximum $\Gamma_{\beta} \Gamma_{\gamma}$. We find $\delta P_{\beta \|} / \delta N_{\beta}=\delta P_{\gamma \|} / \delta N$, where $\delta P_{\beta \|}$ and $P_{\gamma \|}$ are components along $\bar{r}_{\beta}-\bar{r}_{\gamma}$. This represents rigid-body kinematics equivalent to (Landau 1969, Diu et al 1990, Sanmartín 1995)

$$
\delta \bar{P}_{\alpha} / m \delta N_{\alpha}=\omega \wedge \bar{r}_{\alpha} \quad(\bar{\omega} \text { independent of } \alpha) .
$$

We also find $T$ and $\mu-\frac{1}{2} m(\bar{\omega} \wedge \bar{r})^{2}$ uniform. Since the equilibrium angular velocity $\bar{\omega}$ must be timeindependent, it must lie along a principal axis of inertia in order to avoid precession. If $I_{A}$ is the moment of inertia, we have $\bar{L}_{A *}=I_{A} \bar{\omega}$ and

$$
E_{*}=E_{i}+L_{A *}^{2} / 2 I_{A} \text {. }
$$

For solids (and liquids) which have energy density large compared to pressure, mass and energy densities will keep nearly uniform even for velocities $\bar{\omega} \wedge \bar{r}_{\alpha}$ comparable to thermal velocities. The equilibrium entropy will then be

$$
\begin{aligned}
\ln v_{e} & \simeq \sum_{\alpha} \delta N_{\alpha} \ln f\left(V / N, E_{i} / N\right) \\
& =\ln \Gamma\left(N, V, E_{*}-L_{A *}^{2} / 2 I_{A}\right) .
\end{aligned}
$$

For $\ln v_{e}$ to be maximum, $I_{A}$ should be the highest moment of inertia.

Note that ignoring conservation of $\bar{L}_{A *}$ would be valid for $\bar{L}_{A *}=0$. Actually, pressure breaks the conservation of angular momentum, as it broke that of momentum, justifying in this way our original disregard of it; pressure was then found to be uniform at equilibrium, thus exerting no net moment on the surface of the body, and preserving a vanishing $\bar{L}_{A *}$ value. There are instances, however, where the external pressure has negligible effect during the approach to equilibrium as regards momentum and angular momentum (approach to rigid-body motion). This is the case of gravitationally bound systems of bodies (Sanmartín 1995). It is often the case for the motion of systems of solid (or solid and liquid) bodies through a medium like air, drag effects vanishing with the air to solid density ratio. (At high Reynolds number, the relative change of momentum of a solid, as it moves through the air over a distance comparable to its own length, is of the order of the density ratio).

(iv) Equation (9) shows that $\mu$ (chemical potential) is equivalent to energy per particle of a potential field caused by an external, faraway source, the field thus being (nearly) uniform throughout the body. If any such real potential $\phi$ is actually present, equation (9) remains valid if just a term $\phi \mathrm{d} N$ is added to its righthand side. Note, however, that if $N$ (and $V$ and $E_{i}$ ) were fully constant, the body would be a closed system despite the external field, which would have no effect because of its spatial uniformity. On the other hand, for a nearby source, $\phi$ will vary across the body though not across a subsystem, the body being no longer a closed system. Equation (4) may again be used to deal with this problem, the fixed-energy condition now applying to the quantity

$$
\begin{aligned}
\delta E_{i \beta} & +\delta E_{i \gamma}+\delta P_{\beta}^{2} / 2 m \delta N_{\beta}+\delta P_{\gamma}^{2} / 2 m \delta N_{\gamma} \\
& +\phi\left(\bar{r}_{\beta}\right) \delta N_{\beta}+\phi\left(\bar{r}_{\gamma}\right) \delta N_{\gamma} .
\end{aligned}
$$

There is no fixed-momentum condition, the potential barrier being introduced $a b$ initio to avoid wholebody displacement caused by the field. One then finds $\delta \bar{P}_{\alpha} / \delta N_{\alpha}$ vanishing, and $T$ and $\mu+\phi$ uniform. (To prove this last result, Landau and Lifshitz (1969) considered not any two subsystems but two neighbouring parts, so as to allow direct exchange of particles. Note, however, that those parts must be subsystems for $\phi$ to take definite values in each, and need not be neighbours (the particle-exchange need not be direct) for the argument to apply; further, a result 
$\mu_{\beta}+\phi_{\beta}=\mu_{\gamma}+\phi_{\gamma}$ yields nothing new for neighbour subsystems, which have a common location (section 2).) Since only one intensive quantity, $T$, is uniform, the equilibrium entropy

$$
\ln v_{e} \simeq \sum_{\alpha} \delta N_{\alpha} \ln f\left[\frac{\delta V}{\delta N}\left(T, \mu_{\alpha}\right), \frac{\delta E_{i}}{\delta N}\left(T, \mu_{\alpha}\right)\right]
$$

cannot be written as a single function $\ln \Gamma$ for the entire body, although $\ln \Gamma\left(\delta N_{\alpha}, \delta V_{\alpha}, \delta E_{i \alpha}\right)$ remains as entropy of an element $\alpha$.

\section{Conclusions}

Using the detailed macroscopic description of continuum mechanics, and the bare concept of microstate, we were able to derive the principles of thermodynamics from mechanics. In contrast to statistical physics, our derivation laid emphasis on a definite prescription for macrostates (and non-equilibrium entropy). We used basic features of the macrostate description: The description involves either conservative and additive quantities or densities, and is thus scale-free; average densities are kept fixed in the thermodynamic limit; the macrostate concept refers to finite velocities and distant regions, and thus introduces time indirectly. No ergodic-type considerations were involved in the derivation.

\section{Acknowledgments}

This work was supported by the Comision Interministerial de Ciencia y Tecnología of Spain (Project ESP920989-E).

\section{References}

Baierlein R 1994 Am. J. Phys. 62 15-26

Batchelor G K 1967 An introduction to fluid dynamics

(Cambridge: Cambridge University Press)

Bauman R P 1992 Modern Thermodynamics with Statistical Mechanics (New York: McMillan)

Callen H B 1974 in Foundations of Continuum

Thermodynamics ed J J Delgado Domingos, M N R Nina and J H Whitelaw (London: MacMillan) 61-78

Diu B, Guthmann C, Lederer D and Roulet B 1990 Am. J. Phys. 58 974-8

Grandy W T Jr 1987 Foundations of statistical mechanics I (Reidel: Dordrech)

Gyftopoulos E P and Beretta G P 1991 Thermodynamics.

Foundations and applications (New York: MacMillan)

Khinchin A I 1949 Mathematical foundations of statistical mechanics (New York: Dover) section 13

Landau L D and Lifshitz EM 1969 Statistical Physics 2nd edn (Oxford: Pergamon) ch 1 and 2

Lewis J D (ed) 1985 Teaching Thermodynamics (New York: Plenum)

Sanmartín J R 1995 Eur. J. Phys. 16 8-13

Truesdell C 1969 Rational Thermodynamics (New York:

McGraw-Hill) 